\title{
Atomic and molecular LIBS spectra of Al alloys and composite materials for nanosecond and microsecond excitation
}

\author{
R. Ostrowski, W. Skrzeczanowski, A. Rycyk, K. Czyż, A. Sarzyński, M. Strzelec, ${ }^{*}$ K. Jach, R. Świerczyński \\ Institute of Optoelectronics, Military University of Technology, Gen. Sylwestra Kaliskiego 2, 00-908 Warszawa
}

Received August 29, 2017; accepted December 14, 2017; published December 31, 2017

\begin{abstract}
Spectral investigations in the UV-VIS range of selected Al alloys and composites, used in the construction of armament components as well as drones, were performed using the LIBS technique. Two Nd:YAG lasers were applied: a short pulse $4 \mathrm{~ns} 60 \mathrm{~mJ}$ laser and a long pulse 200/400 (up to 1000) $\mu$ s ( 2/4 up to $10 \mathrm{~J}$ ) laser. In experiments with a short laser pulse only atomic spectra were observed while for long pulses, also molecular transitions have been recorded. Electron temperatures of created plasma were found on the base of Boltzmann law. Temperatures are clearly higher for short pulse excitation.
\end{abstract}

Laser Induced Breakdown Spectroscopy (LIBS) is a powerful technique used to determine the chemical composition and analyze the structures of any solid, liquid or gas objects. The LIBS method consists in evaporating (using high power/energy laser pulse) a small amount of analyzed material and creating a plasma cloud emitting continuous and linear radiation. The analysis of linear plasma radiation allows identification of elements existing in the sample, determination of their concentrations as well as evaluation of stratigraphic characteristics of surface object layers [1].

In the presented experiments, two types of $\mathrm{Nd}$ :YAG laser were utilized for excite of a plasma cloud on the surface of selected materials. The first Nd:YAG laser worked in the free running regime, and emitted long 400 (200) $\mu$ s pulses with energies of $4 \mathrm{~J}(1064 \mathrm{~nm})$. The second, Q-switched Nd:YAG laser generated energies up to $60 \mathrm{~mJ}$ in $4 \mathrm{~ns}$ pulses on a wavelength of $1064 \mathrm{~nm}$, Plasma radiation was recorded in the UV-VIS region $(200 \div 780 \mathrm{~nm})$ with a spectral resolution of 20000 by an ESA 4000 spectrometer. Spectra were recorded from one laser shot. The registration time window was equal to the time duration of a laser pulse in the case of the free running laser, and $1 \mu \mathrm{s}$ in the case of the Q-switched laser. The spectra were recorded in air under atmospheric pressure.

The measurements of UV-VIS spectra were carried out on aluminum, aluminum alloys and composites. These materials are commonly used in the construction of different armament components as well as drones. The results of analyses include plasma radiation spectra, their comparison using multivariant statistical analysis and determination of plasma temperatures.

\footnotetext{
*E-mail: marek.strzelec@wat.edu.pl
}

The analyses were performed on technically pure aluminium and on $\mathrm{Al}$ alloys used in the construction of shoulder-launched, anti-tank rocket-propelled grenade type of RPG-7 and 60mm mortar projectile.

Figures 1-2 present exemplary emission spectra of investigated materials with an indicated majority of identified transitions (spectral lines) [2]. In the case of spectra recorded using long laser pulse $4 \mathrm{~J}, 400 \mu \mathrm{s}$, electronic transitions, recorded as single spectra lines were supplemented by vibrational-rotational bands (Figs. $3-4)$.

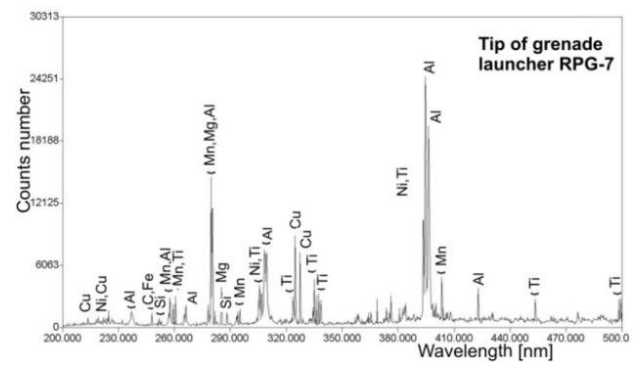

Fig. 1. Emission spectrum of tip of grenade RPG-7 recorded using short pulse laser $60 \mathrm{~mJ} / 4 \mathrm{~ns}$ (time gate $1 \mu \mathrm{s}$, delay from laser pulse $500 \mathrm{~ns}$ ).

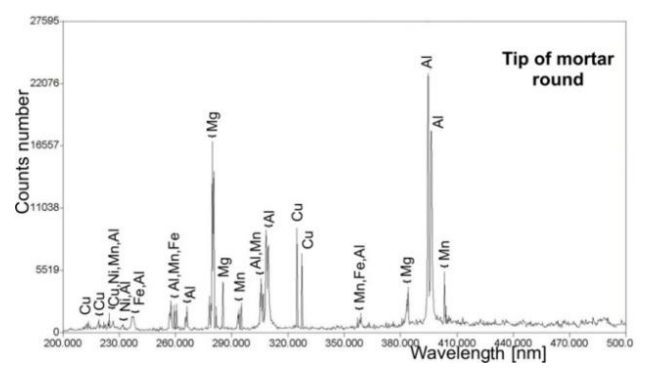

Fig. 2. Emission spectrum of the tip of a $60 \mathrm{~mm}$ mortar round taken with a short pulse laser $60 \mathrm{~mJ} / 4 \mathrm{~ns}$ (time gate $1 \mu \mathrm{s}$, delay from laser pulse 500ns).

The spectrum of technically pure aluminum is not very complex and contains a small amount of lines, primarily $\mathrm{Al}$ lines. On the other hand, the fundamental difference, which can be seen in the spectra of elements of RPG-7 grenade and mortar projectile, is the presence of different alloying components. The grenade, except common alloying admixtures, like $\mathrm{Fe}, \mathrm{Cu}$ and $\mathrm{Mg}$, contains a large amount of titanium, while in the emission spectrum of the tip of a mortar round are visible the lines of $\mathrm{Mn}$ and $\mathrm{Ni}$ (Fig. 2). 


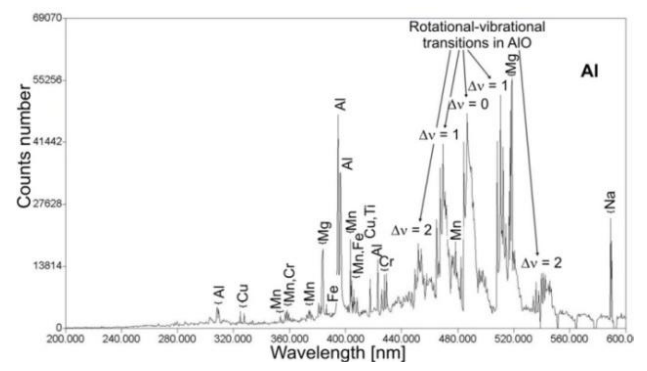

Fig. 3. Emission spectrum of technically pure aluminum recorded using a long pulse laser $4 \mathrm{~J}$ and $400 \mu$ s (time gate $400 \mu \mathrm{s}$ ).

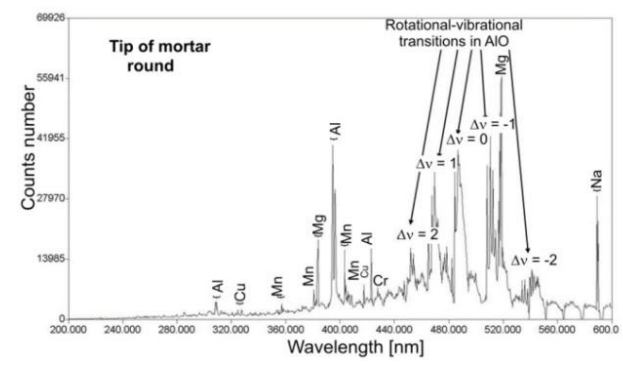

Fig. 4. Emission spectrum from the tip of a $60 \mathrm{~mm}$ mortar projectile recorded using a long pulse laser $4 \mathrm{~J}$ and $400 \mu$ s (time gate $400 \mu \mathrm{s}$ ).

The spectra of $\mathrm{Al}$ and $\mathrm{Al}$ alloys recorded with a long pulse laser (Figs. 3-4) show clear differences in comparison with the spectra presented in Figs. 1-2 taken for short delays from a laser pulse (500ns). On the other side, although they both seem to be very similar for long pulse excitation, the application of a multivariant statistical analysis [3, 4], factorial analysis, allows fast identification of similarities and differences in the composition of analyzed materials, not requiring a laborious analysis of individual spectral lines. The example of a factorial analysis of $\mathrm{Al}$ samples is shown in Fig. 6.

Definitely clearer spectra differences are observed in comparison to irradiation with short (nanosecond) and long (microsecond) laser pulses. It is shown in Fig. 4 for Al alloy in a mortar projectile. The fundamental differences consist in a significantly weaker input of the UV part of spectra and the presence of bands corresponding to the vibrational-rotational transitions of aluminum oxide $\mathrm{AlO}$ molecules in the case of irradiation with a long laser pulse. AlO molecules occur due to the interaction of plasma from an $\mathrm{Al}$ target with the surrounding atmosphere. This interaction is several orders of magnitude longer than in the case of a short laser pulse. Moreover, molecular transitions are recorded easier in plasmas with a lower temperature.

The role of the plasma-environment interaction length was also confirmed in the case of the short-pulse laser, for which we recorded spectra for a longer time window and higher delay from a laser pulse. However, the spectral signal from molecular transitions in this case was much smaller and we needed 50-100 laser shot accumulations to record one useful spectrum (atomic lines were overexposed in this case).

Some attempts were made to find the time interval in which molecular transitions occur. This was obtained by scanning $4 \mathrm{~ns}$ laser generated plasma with $10 \mu$ s steps and $10 \mu$ s time gate, which is shown in Fig. 6. The results are in reasonable accordance with the data presented in [5].

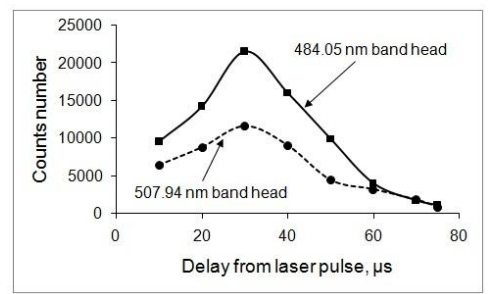

Fig. 5. Temporal behaviour of vibrational-rotational transitions in an AlO molecule. The data was drawn from spectra taken with $10 \mu$ s time gates at $10 \mu \mathrm{s}$ steps of delay from $4 \mathrm{~ns} 60 \mathrm{~mJ}$ laser pulse.

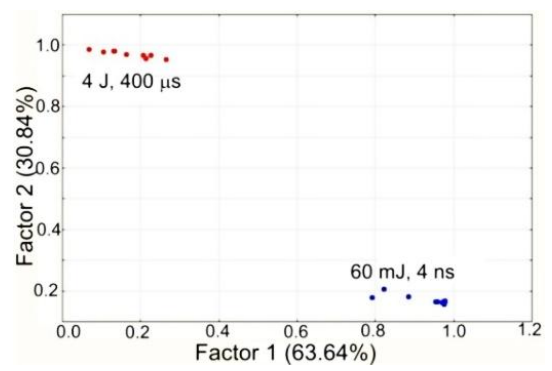

Fig. 6. Factorial comparison of LIBS spectra of a mortar projectile recorded using a short $(4 \mathrm{~ns})$ and long $(400 \mu \mathrm{s})$ laser pulse

Analyses were performed for three samples of unknown composites, called in the text conventionally according to their colors. An example of the LIBS spectrum of black composite is shown in Fig. 7.

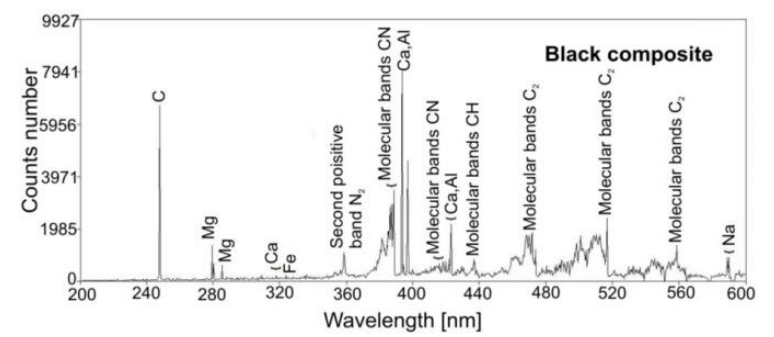

Fig. 7. LIBS spectrum of black composite recorded using laser $60 \mathrm{~mJ}$ and 4 ns.

The white-yellow and dark-yellow composites, similarly like composites discussed in the literature [6,7], differ mainly due to the content of additives, like boron, magnesium, silicon, calcium, iron, aluminum and titanium. What is characteristic is the lack of vibrationalrotational bands, which are clearly visible, except sharp spectral lines of electronic transitions in the LIBS spectrum of a black composite in Fig. 7. Molecular bands in spectra from the black composite were observed both for short $(1 \mu \mathrm{s})$ and long $(100 \mu \mathrm{s})$ time gates.

The spectrum of the black composite contains a very strong line of neutral carbon CI $247.8 \mathrm{~nm}$, as well as the 
vibrational-rotational transitions in $\mathrm{N}_{2}$ (358nm), $\mathrm{C}_{2}$ (516nm), CN (388nm) and $\mathrm{CH}$ (438nm) molecules.

The LIBS spectra of all composite samples recorded during the experiment with a long laser pulse practically do not allow the identification of any transition or spectral lines. They are typical of radiation with a continuum spectrum.

The factorial analysis of the LIBS spectra of composites is presented in Fig. 8. The comparison of composites spectra for two time durations of laser pulses show that the time of excitation determines primarily their similarities. The spectra of all composites for a long laser pulse are almost identical and clearly differ from their analogues recorded using a short laser pulse.

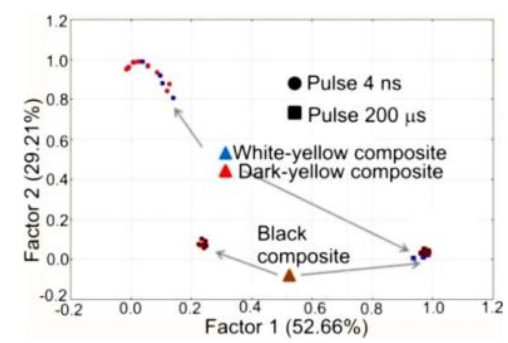

Fig. 8. Comparative factorial analysis of LIBS spectra of composites recorded using different duration time of pulses.

The recording of LIBS spectra of different materials made it possible to determine of the recorded plasma temperatures. Boltzmann's plots were utilized to determine the excitation temperature of energetic levels responsible for transitions recorded as spectral lines. The fundamental assumption of this method is the so-called LTE balance (local thermodynamic equilibrium), in which the population of energetic levels in atoms and ions results from the reciprocal interaction of atoms and ions with electrons, characterized by Maxwell's velocity distribution. The density of a radiation field can be neglected in this method.

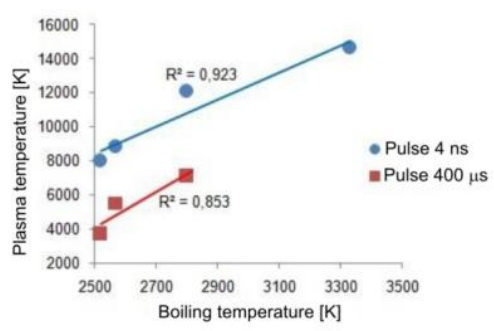

Fig. 9. Plasma excitation temperature as a function of material boiling temperature.

An example of resulting plasma temperatures for short and long excitation pulses is presented in Fig. 9. The course of plasma temperature changes shows its dependence on the boiling temperature of an irradiated material. Several researchers observed that some sample properties, like melting and boiling temperatures, hardness or microhardness correlate with plasma temperature and other plasma characteristics [8-10] or not [11], which needs further studies.

In conclusions, the spectroscopic UV-VIS investigations of $\mathrm{Al}$ alloys and selected composites used in the elements of military technology were performed using the LIBS method with two laser sources: $60 \mathrm{~mJ}, 4 \mathrm{~ns}$ and $4 \mathrm{~J}, 400 \mu \mathrm{s}$ $(200 \mu \mathrm{s})$. These LIBS spectra, recorded in the range of $200 \div 780 \mathrm{~nm}$ were achieved for each material. It allowed qualitative determination of chemical composition of analysed samples and their similarities with the aid of statistical treatment and analysis. During the irradiation of Al alloys with long laser pulses, the LIBS spectra have shown also vibrational-rotational $\mathrm{AlO}$ bands, which resulted from longer interaction of excited plasma with the atmosphere. These molecular bands were also observed for short pulse laser irradiation, but for long delays from the laser pulse $(10-80 \mu \mathrm{s})$. Due to cold plasma in this time interval, spectra recording needed 50-100 accumulations.

Plasma temperatures, determined for different materials using Boltzmann's plots, are substantially higher in the case of plasma excited by a short laser pulse, which is the result of much higher radiation power density. The observed dependence of plasma temperature on the boiling temperature of analysed materials needs continuation of similar research including a wider range of laser parameters as well as a broader spectrum of materials.

The work was performed as part of project No DOB-16/1/PS/2014, funded by the National Centre for Research and Development in Poland, entitled "Laser Systems of Directed Energy Weapons, Laser Systems of Non-Lethal Weapons".

\section{References}

[1] A. Miziolek, V. Palleschi, I. Schechter, Laser-Induced Breakdown Spectroscopy (Cambridge, Cambridge University Press 2006).

[2] M. Sabsabi, P. Cielo, Appl. Spectrosc. 49, 499 (1995).

[3] D.L. Death, A.P. Cunningham, L.J. Pollard, Spectrochim. Acta B 63, 763 (2008).

[4] S. Laville, M. Sabsabi, F. Doucet, Spectrochim. Acta B 62, 1557 (2007).

[5] D.M. Surmick, C.G, Parigger, Applied Spectroscopy 68(9), 992 (2015).

[6] J.B. Sirven, B. Bousquet, L. Canioni, L. Sarger, Anal. Chem. 78, 1462 (2006).

[7] E.J. Judge, G. Heck, E.B. Cerkez, R.J. Levis, Anal. Chem. 81, 2658 (2009).

[8] L.M. Cabalin, J.J. Laserna, Spectrochim. Acta B 53, 723 (1998).

[9] T.A. Labutin, A.M. Popov, V.N. Lednev, N.B. Zorov, Spectrochim. Acta B 64, 938 (2009).

[10] J.S. Cowpe, R.D. Moorehead, D. Moser, J.S. Astin, S. Karthikeyan, Spectrochim. Acta B 66, 290 (2011).

[11] G. Cristoforetti, S. Legnaioli, V. Palleschi, A. Salvetti, E. Tognoni, Spectrochim. Acta B 63, 312 (2008). 